\title{
Entransy and entropy revisited
}

\author{
HU GuoJie, CAO BingYang \& GUO ZengYuan* \\ Key Laboratory for Thermal Science and Power Engineering of Ministry of Education School of Aerospace, Tsinghua University, Beijing 100084, \\ China
}

Received January 21, 2011; accepted May 18, 2011

\begin{abstract}
Entransy, a recently developed concept, is the central physical quantity characterizing heat transfer processes not related to heat-to-work conversions. The entransy of an object pertains to the nature of the potential energy of heat in a thermal field and describes its heat transfer ability. In the present study, we revisit this concept, and develop its relationship to state and process quantities. This then enables a direct comparison to the more familiar concept, entropy, the central physical quantity in thermodynamics. The comparison helps to identify the role entransy has in heat transfer processes and highlight under what conditions state and process quantities related to entransy can be distinguished in such processes. As to embody the entransy loss due to work expended between the system and its environment for the irreversible heat conduction in gases, new quantities, available entransy flow and available system entransy are introduced. Both the entransy of solids and the available system entransy of gases are state quantities and their changes correspond to the entransy flow and the available entransy flow respectively. Thus there is no need to stress the difference between process quantity and state quantity in heat transfer.
\end{abstract}

entransy, available entransy, state quantity, process quantity

Citation: $\quad$ Hu G J, Cao B Y, Guo Z Y. Entransy and entropy revisited. Chinese Sci Bull, 2011, 56: 2974-2977, doi: 10.1007/s11434-011-4582-0

Entransy [1] is a new physical quantity characterizing heat transfer that pertains to the nature of potential energy and describes heat transferability of a system. Based on the thermomass theory, entransy represents the potential energy of thermomass [2,3]. Entropy, in addition to energy, is a central physical quantity in thermodynamics. It is a measure of the irreversibility of a process and a criterion describing the thermal equilibrium of a system. It also represents the degree of disorder within a system. The minimum principle of entropy generation is also an important criterion in process optimization. Because of its general features, the entropy concept has been extended to many other fields, such as thermodynamic optimization, quantum theory, and information theory [4]. These extended applications have already lead to great advancements, but doubts have arisen. For example, in the optimization of heating or cooling devices, minimum entropy generation does not always correspond to best performance [5-7]. To address this discrep-

*Corresponding author (email: demgzy @tsinghua.edu.cn) ancy, Guo et al. proposed a new physical quantityentransy [1]. Based on this concept, it is now possible to characterize heat transfer efficiency. Taking the entransy concept a step further, entransy dissipation, a measure of heat transfer irreversibility, forms the basis for a new principle of least action called the extremum principle of entransy dissipation [8]. These concepts have successfully been used in the optimization of heat conduction $[9,10]$, heat convection $[11,12]$ and heat radiation $[13,14]$.

Comparing entransy and entropy, two questions necessarily arise. First, is entransy a state quantity? Second, are there differences between the state quantity and the process quantity in heat transfer? If there are, then why have these two heat transfer quantities never been distinguished?

\section{Is entransy a state quantity?}

\subsection{System entransy}

In [1], the entransy of an object is identified as 


$$
G=\frac{1}{2} U T,
$$

where $T$ is the temperature, and $U$ the internal energy of a system. Because volume changes are not usually considered in heat transfer, eq. (1) represents the entransy of a system under constant volume. As $T$ and $U$ are both state quantities, this expression is obviously a state quantity. However, this form of the definition is different from that for entropy in two aspects: (1) the definition of entropy is in a differential form; and (2) entropy is defined by a relationship involving state and process quantities. To make a more direct comparison, entransy needs to be defined in a similar form to entropy. The concept of entransy for this reason needs to be revisited.

\subsection{Clausius entropy and its improvement}

Clausius defined entropy [15] as the quotient of the process quantity, $\delta Q$, and the state quantity, Kelvin temperature $T$, in a reversible process

$$
\left(\frac{\delta Q}{T}\right)_{\text {rev }}=\mathrm{d} S,
$$

where $S$ is the system entropy $\delta Q / T$ is the entropy flow, and the subscript 'rev' signifies the quantities related to a reversible process.

$\mathrm{Wu}$ [16] extended this definition to include changes in entropy associated with work

$$
\left(\frac{\delta Q}{T}\right)_{\text {rev }}=\mathrm{d} S=\mathrm{d} S_{\mathrm{u}}+\mathrm{d} S_{\mathrm{w}}=\frac{\mathrm{d} U+p \mathrm{~d} V}{T},
$$

where $\mathrm{d} S_{\mathrm{u}}=\mathrm{d} U / T$ is the quotient of the internal energy and temperature, and $\mathrm{d} S_{\mathrm{w}}=p \mathrm{~d} V / T$ is that of the work output and temperature; these terms are both related to state quantities of a system.

As $\delta Q$ is a process quantity, its product with the state quantity $1 / T$ should essentially be a process quantity. However, only in reversible processes does the entransy flow, $(\delta Q / T)_{\text {rev }}$, correspond to a state quantity of a system. In that case, $(\delta Q / T)_{\text {rev }}$ can be identified as an exact differential $\mathrm{d} S$ of the state quantity $S$ of the system.

\subsection{Does the entransy flow correspond to a state quantity of a system?}

The entransy flow is defined as the product of heat and temperature under reversible conditions

$$
(T \delta Q)_{\mathrm{rev}}=\delta G^{*}
$$

Whether the entransy flow, $G^{*}$, corresponds to the state quantity of a system is unknown. Here we use the symbol " $\delta$ " rather than "d" to indicate that $\delta G$ " may be an inexact differential.

For a system not changing volume, the first law of thermodynamics leads to

$$
(\delta Q)_{\text {rev }}=\mathrm{d} U
$$

Multiplying eq. (5) by temperature, $T$, results in

$$
(T \delta Q)_{\text {rev }}=\delta G^{*}=T \mathrm{~d} U=\mathrm{d} G .
$$

Eq. (6) tells us that under reversible conditions the entransy flow in the absence of volume change corresponds to a state quantity of the system. In this case, $G$ is called the system entransy.

For a system undergoing volume change, the first law of thermodynamics, however, leads to

$$
\delta Q=\mathrm{d} U+\delta W
$$

and the ideal gas equation of state gives

$$
P V=n R T \text {. }
$$

Multiplying eq. (7) by temperature, $T$, and substituting eq. (8) into eq. (7) yields

$$
(T \delta Q)_{\mathrm{rev}}=T \mathrm{~d} U+T \delta W=T \mathrm{~d} U+T^{2} \frac{n R}{V} \mathrm{~d} V
$$

where $p, V, T, n$, and $R$ are the pressure, volume, temperature, molar quantity, and gas constant of the system, respectively; $U$ is the internal energy of the system, and $W$ is the process work.

Because the last term of eq. (9) cannot be simplified as a single-valued function of certain state quantity, the process quantity $(T \delta Q)_{\text {rev }}$ does not correspond to a state quantity of the system and thus cannot be identified as such.

\section{Available entransy}

\subsection{Available energy (exergy) in thermodynamics}

In thermodynamics, available energy can be divided into the available energies of heat and of the system. For heat, $Q$, its maximum capacity of doing work at ambient temperature, $T_{0}$, is defined as its available energy, that is heat exergy $[16,17]$

$$
E_{\mathrm{x}, \mathrm{Q}}=W_{\max }=Q\left(1-\frac{T_{0}}{T}\right),
$$

where $T$ is the temperature of the heat source from which the heat is removed, and $E_{\mathrm{x}, \mathrm{Q}}$ is the heat exergy.

For a thermodynamic system (i.e. a closed system) changing under some reversible processes from a certain state to an equilibrium state within an environment, its maximum capacity for doing work is defined as its available energy, that is the system exergy $[16,17]$

$$
E_{\mathrm{x}, \mathrm{U}}=W_{\mathrm{u}, \max }=U-U_{0}-T_{0}\left(S-S_{0}\right)+p_{0}\left(V-V_{0}\right),
$$


where $\left(p_{0}, T_{0}, V_{0}, U_{0}, S_{0}\right)$ is the state of the environment, and $(p, T, V, U, S)$ is the initial state. Therefore, the system exergy is a state quantity of the combined system (i.e. system and environment).

\subsection{Available energy and available entransy in heat transfer}

(i) Available energy of heat for object heating. In a heat-to-work conversion process, only a part of the input heat can be used to do work. The available energy of heat (i.e. conversion available energy) is identified as its maximum capacity of doing work (i.e. the work output in a Carnot cycle). Similarly, within thermodynamics, the concept of available energy in heat transfers also exists. For a system at constant volume, the input heat can be used to increase the system temperature and equals the change in the internal energy of a system. For a system changing volume, the input heat cannot all be used to increase the temperature of a system if there is work exchange with the outside. However, the maximum energy that can be used to heat objects equals the input heat itself (such as in an isochoric process); that is, all the input heat can be used to heat objects. This means the available energy in a heat transfer is one hundred percent of the heat and corresponds to the change in the internal energy of a system undergoing an isochoric process. Therefore, we have

$$
(\delta Q)_{\text {rev,ava }}=(\mathrm{d} U)_{\mathrm{V}} .
$$

(ii) Available entransy. For a system for which volume can change, a portion of the entransy flow will be converted into work entransy, $T \delta W$, given in eq. (9), which makes no contribution to the heating or cooling of an object. $T \mathrm{~d} U$ is the available portion of the entransy flow, i.e. the available entransy flow. Hence, we have

$$
(T \delta Q)_{\text {rev,ava }}=T \mathrm{~d} U=\mathrm{d} G_{\text {ava }} .
$$

Eq. (13) shows that the available entransy flow can be associated with a state quantity of system, $G_{\text {ava }}$, which is identified as the available entransy of the system. In thermodynamics, the maximum capacity for doing work is identified as the system exergy, also called the available energy for heat-to-work conversion, whereas in heat transfer the maximum capacity of transferring heat is identified as the available entransy, and is similarly called the available entransy for heat transfer.

\section{Process quantity and state quantity}

In thermodynamics, it is a requirement to distinguish the state quantity from the process quantity, because in the thermodynamic cycle for heat-to-work conversion with a gas as medium, two process quantities, i.e. heat and work, correspond to one state quantity of the system whereas those two process quantities are distinct and cannot be directly summed. Thus, under normal circumstances, the change in a certain process quantity does not equate to the change in state quantity of the system; hence, it is necessary to distinguish the state quantity and process quantity in heat-to-work conversion processes.

In a heat transfer process, however, we do not have to consider changes in the system volume. Therefore, the state quantity of the system only corresponds to a certain process quantity; that is, a change in the process quantity equates to an equal change in the corresponding state quantity. Hence, distinguishing state and process quantities is unnecessary. Similarly, for a heat transfer process in which the volume of the medium (i.e. system) changes, the applied work resulting from volume change makes no contribution to the system's capacity in heating or cooling objects, whereas only the available portion of heat and entransy should be considered. Under reversible conditions, the available heat energy and available entransy flow correspond to the internal energy and available entransy of system, respectively. Thus, it is not necessary to distinguish the state and process quantities.

\section{Conclusions}

(1) In heat transfers, differences between state quantity and process quantity exist. The entropy flow, $\delta Q / T$, under reversible conditions is essentially a process quantity; however, it corresponds to a state quantity of the system and can be identified as the system entropy $S$. The entransy flow, $T \delta Q$, is a process quantity. For heat conduction in the absence of volume change under reversible conditions, it also corresponds to a state quantity and can be identified as the system entransy, $G$.

(2) New physical quantities, viz. heat transfer available energy and available entransy, are identified. For gases, heat and entransy flow do not correspond to state quantities even under reversible conditions, but the available energy of heat transfer and available entransy flow correspond to the change in the internal energy and available system entransy, respectively.

(3) In heat transfer processes, only available heat and available entransy need to be considered; both correspond to state quantities of a system. Therefore, it is unnecessary to distinguish the state quantity and process quantity.

This work was supported by the Tsinghua University Initiative Scientific Research Program.

1 Guo Z Y, Zhu H Y, Liang X G. Entransy-a physical quantity describing heat transfer ability. Int J Heat Mass Transfer, 2007, 50: 2345-2556

2 Cao B Y, Guo Z Y. Equation of motion of a phonon gas and non- 
Fourier heat conduction. J Appl Phys, 2007, 102: 053503

3 Guo Z Y, Cao B Y. A general heat conduction law based on the concept of motion of thermal mass (in Chinese). Acta Phys Sin, 2008, 57: 4273-4280

4 Shannon C E. A mathematical theory of communication. Bell Syst Tech J, 1948, 27: 379-423, 623-656

5 Liu X B, Meng J A, Guo Z Y. Entropy generation extremum and entransy dissipation extremum for heat exchanger optimization. Chinese Sci Bull, 2009, 54: 943-947

6 Guo Z Y, Liu X B, Tao W Q, et al. Effectiveness-thermal resistance method for heat exchanger design and analysis. Int $\mathrm{J}$ Heat Mass Transfer, 2010, 53: 2877-2884

7 Liu X B, Guo Z Y. A novel method for heat exchanger analysis (in Chinese). Acta Phys Sin, 2009, 58: 4766-4771

8 Cheng X G. Entransy and Its Applications in Heat Transfer Optimization (in Chinese). Beijing: Tsinghua University, 2004. 39-44

9 Guo Z Y, Cheng X G, Xia Z Z. Least dissipation principle of heat transport potential capacity and its application in heat conduction optimization. Chinese Sci Bull, 2003, 48: 406-410

10 Chen L G, Wei S H, Sun F R. Constructal entransy dissipation mini- mization for 'volume-point' heat conduction. J Phys D: Appl Phys, 2008, 41: 195506

11 Chen Q, Wu J, Ren J X. Thermodynamic and heat transfer optimization of convective heat transfer (in Chinese). J Eng Thermophys, 2008, 29: 271-274

12 Chen Q. Irreversibility and Optimization of Convective Transport Processes (in Chinese). Beijing: Tsinghua University, 2008

$13 \mathrm{Wu}$ J, Liang X G. Application of entransy dissipation extremum principle in radiative heat transfer optimization. Sci China Ser ETech Sci, 2008, 51: 1306-1314

14 Cheng X T, Liang X G. Entransy flux of thermal radiation and its application to enclosures with opaque surfaces. Int J Heat Mass Transfer, 2011, 54: 269-278

15 Clausius R. Über die bewegende Kraft der Wärme, Part I, Part II (in German) I. Annalen der Physik, 1850, 79: 368-397, 500-524

$16 \mathrm{Wu}$ J. Potential energy (Entransy) in thermal science and its application (in Chinese). Disseration for the Doctoral Degree. Beijing: Tsinghua University, 2009. 82-83, 107-111

17 Shen W D, Jiang Z M, Tong J G. Engineering Thermodynamics (in Chinese). 3rd ed. Beijing: Higher Education Press, 2001

Open Access This article is distributed under the terms of the Creative Commons Attribution License which permits any use, distribution, and reproduction in any medium, provided the original author(s) and source are credited. 